\title{
An Analytical Model for Deploying Mobile Sinks in Industrial Internet of Things
}

\author{
Maryam Vahabi*, Hamid Reza Faragardi ${ }^{\dagger}$, Hossein Fotouhi* \\ *School of Innovation, Design and Technology / Mälardalen University, Västerås, Sweden \\ \{maryam.vahabi, hossein.fotouhi\}@mdh.se \\ ${ }^{\dagger}$ Department of Theoretical Computer Science, KTH Royal Institute of Technology, Stockholm, Sweden \\ hrfa@kth.se
}

\begin{abstract}
Nowadays, the Industrial Internet of Things (IIoT) has the potential to be implemented in factories and supply chains to improve manufacturing efficiency. It is becoming more common to use mobile robots in such factories for further improvements. Adding data collection capability to the mobile robots would realize the mobile sink deployment in future factories. As it is important to reduce the deployment cost, we are aiming at a network with minimum number of mobile sinks while ensuring network reliability and timeliness. In this paper, we analytically model a given trajectory for the motion of mobile sinks and the routing of mobile sinks along the trajectory in an IIoT system. We introduce an optimization problem in the form of Integer Linear Programming (ILP) to specify the minimum number of required mobile sinks to reduce deployment cost of an IIoT system, and also to identify the routing of multiple mobile sinks along a given trajectory. The proposed ILP model can be solved by several existing off-the-shelf ILP-solvers.
\end{abstract}

\section{INTRODUCTION}

The industrial trends and initiatives are aiming at smart factory, where all industrial equipments and production lines are supposed to be connected to each other through Internet [1]. IIoT is able to provide continuous monitoring of devices, while predicting and reporting problems and failures, which in turn provides more efficient production, and avoids hazards in factories. Wireless Sensor Networks (WSNs) are the key enablers for IIoT systems by providing a wireless connection between sensors and actuators.

Traditionally, WSNs with static nodes formed a tree-based routing strategy in order to transmit measurements toward the destination [2]. Some of the consequences of collection tree routing protocols at nodes in the vicinity of destination are packet collision and network disconnectivity that lead to packet re-transmissions and consequently longer delays. This problem has been tackled in various research works by applying contention-based [3] or scheduling-based [4] mechanisms.

A solution for data collection in networks with fixed sensor nodes that have limited energy resources is to employ mobile sinks endowed with much higher energy capacity [5]. In this case, sink mobility increases network lifetime by providing the opportunity of having several collection points. Thus, it is less likely to experience high traffic overhead on a number of forwarder nodes toward the sink node.

The use of industrial robots such as Automated Guided Vehicles (AGVs) have increased attention in automation domain [6]. These robots with moving capability are the key enablers of networks with mobile sinks. These robots are expected to visit different places in a factory and collect buffered data from sensors, and then forwarding the data to a Gateway for either local or remote processing on a Cloud platform [7].

Besides the mobile sink deployment, future factories are envisioned to employ Software Defined Networking (SDN) at the sensor network level to bring agility to the network. In order to have a reconfigurable network, SDN controller generates rules for forwarding sensors' data throughout the network [8]. A network with limited resources in terms of energy and bandwidth benefits from the SDN controllers as well. This can be done through tuning sensors' duty cycles and selecting next hop for data transmission. Controllers are supposed to have information exchange with sensors and mobile sinks in order to send appropriate updated rules.

In this work, we focus on a network, where location of sensors and SDN controllers are predefined [7]. We assume two limitations for mobile sinks: (i) mobile sinks can move only along a predefined trajectory. This constraint comes from the space limitation in a factory for the movement of robots carrying the mobile sinks and the robots missions, and (ii) mobile sinks are limited to collect data only on predefined points along the trajectory dubbed as aggregation points, while forwarding the collected data to the Gateway can be performed at any point of the trajectory.

For analogy, imagine the IoT system as a public transport system, where a set of buses (mobile sinks) serve the public transportation. There are a set of predefined bus-stops (aggregation points) to pickup passengers (collecting sensors' data). The first challenge is to specify the location of busstops (aggregation points), wisely such that each passenger (sensor) is located close enough to at least one bus-stop, i.e., the distance between each passenger's location and the bus-stop is not longer than an acceptable distance, which is called $l_{\max }$. The first challenge (i.e., finding optimal location of aggregation points) has been addressed by [7]. The second challenge is to specify the trajectory of mobile sinks such that (i) all the aggregation points are connected, and (ii) the space limitation of the factory for movement of mobile sinks are respected. In this paper, we assume that the trajectory of mobile sinks is predefined, and thus, the second challenge is waived. The third challenge is to find the number of buses (mobile sinks), and the fourth challenge is that in which order the bus-stops (aggregation points) should be traversed by these buses, which is a routing problem. Indeed, the third challenge can not be addressed regardless of the routing of mobile sinks. In other words, the routing information of mobile sinks enables us to decide about the minimum number of mobile sinks. 
For reliability considerations, we expect that in a particular time period, more than one bus (mobile sink) serves each passenger (sensor). The reason is that if the bus crashes, there should be at least one more bus to serve this passenger (sensor). We can generalize this limitation such that each passenger (sensor) should be served by at least $k$ buses (mobile sinks) during a specific time period; this limitation is called $K$-covered constraint.

In such systems, we also encounter timeliness requirement in the sense that we expect the sampled data arrives at the destination no longer than a specific deadline. Two factors play the key role to satisfy the timeliness requirement of such systems: (i) the $l_{\max }$ parameter, and (ii) the maximum workload constraint, which implies that when a bus (mobile sink) reaches a bus-stop (aggregation point), the number of passengers waiting at the bus-stop (total sensors data) should not be more than the capacity of the bus (mobile sink buffer size). There are two options to respect this constraint, which are defined as below:

1) We can assume that the inter-arrival time of buses is identical, meaning that at each bus-stop, the buses arrive periodically, and hence the capacity of each bus should be tuned according to the arrival rate of the passengers in the busiest bus-stop along the trajectory of the bus.

2) We can assume that the capacity of the buses are fixed, and the varying parameter is the inter-arrival rate of two subsequent buses with respect to the arrival rate of passengers to each bus-stop.

In this paper, the latter option is adopted to respect the workload constraint. The optimization problem is to specify the number of mobile sinks and the routing of mobile sinks along the predefined trajectory such that the number of mobile sinks is minimized while ensuring reliability and timeliness. Main contributions of this work are summarized below:

1) Discussing the challenges to employ multiple mobile sinks to provide a promising and practical solution for data collection in IIoT systems.

2) Presenting an analytical model for the deployment of mobile sinks in IIoT, focusing on the routing of multiple mobile sinks in a smart factory.

3) Reducing the deployment cost of IIoT by minimizing the number of required mobile sinks, while respecting network reliability and timeliness.

The paper is organized as follows: in Section II, a comprehensive review of related work is presented. In Section III, we describe the problem and assumptions, which is then followed by our proposed ILP model. Finally, in Section IV, we provide a discussion along with future directions.

\section{RELATED WORK}

In this section, we explain some of the related works on using mobile sinks in IoT applications, which are mainly focusing on energy efficiency issue. Then we will provide some of the main approaches for placing sink nodes in WSNs. In our work, we focus on reducing the number of mobile sinks, and finding their placement in order to reduce the cost of deployment. In turn, it will also lead to lower energy consumption during the run-time as fewer mobile sinks are employed.
Background on employing mobile sink(s) in WSNs. In WSNs with static sinks, nodes located closer to the sinks are more likely to lose their battery power and cause connectivity degradation [9]. Mobile sinks implicitly provide a load balancing paradigm without the need of applying sophisticated routing mechanisms on all nodes, which in fact requires further processing and control message exchanges.

MobiRoute [10] is an all-to-one data collection routing protocol that employs a distance-vector based approach. Route messages are exchanged periodically between neighbors, and the next hop is selected based on a cost function. Three mechanisms are introduced to cope with node mobility. First, to exploit beacon messages and timeouts to detect link breakage due to the moving out of range. Second, to limit the tree reconstruction to reduce the overhead, and tolerate temporary sub optimal routing paths. Third, to use data buffering to mitigate the data loss generated by sink mobility. We are tackling the mobility detection issue by employing an SDN controller with mobility management capability, where it reports nodes where and when to transmit their buffered data.

The Energy Management in a WSN with Multiple Sinks (EMMS) [11] has been proposed to improve the energy consumption. EMMS finds a closed tour of each mobile sink, where the length of the closed tour of all mobile sinks is equal. It also determines the trajectory of each mobile sink on the closed tour. Then builds a routing tree rooted at each sojourn location as well as the sojourn time of the location for the mobile sink. According to the energy level of sensor nodes, routing tree of mobile sinks updates. Unlike EMMS, in this paper, mobility and trajectory of sink nodes is defined in such a way to increase network reliability, while it implicitly holds the energy efficiency issue by considering workloads at sensor nodes.

It is wise to assume some alternative locations for mobile sinks to pause and collect buffered data. Node placement is one of challenging issues in WSNs that would lead to deployment cost reduction by locating sink nodes in proper places, and using minimum number of sinks when sinks are moving. Sensor node placement that targets locating sensors, sinks, and relays can be classified into three groups: (i) exhaustive search algorithms, (ii) discrete optimization-based algorithms, and (iii) approximation-based algorithms. Many research works have been conducted in this field, where some of them are mentioned below.

Background on placement approaches. The exhaustive search or brute-force search is a very general problem-solving technique that considers all possible candidates for the solution, which is obviously time consuming, and thus it is more practical for small scale networks. In [12], the authors proposed two approaches for relay node placement based on exhaustive search, which were called Connected Relay Node Single Cover (CRNSC) and 2-Connected Relay Node Double Cover (2CRNDC). The main idea of the algorithm was based on dividing the network into small cells, and finding the optimal solution within each cell. It also provided connectivity by adding relay nodes in each cell. The controller placement problem for wired networks has been addressed in [13]. They explore the trade-offs when optimizing for minimum latency between nodes and controllers. An extension of [13] that called 
Pareto-Optimal Controller Placement (POCO) [14], considers additional aspects other than network latency. They explored the whole solution space by considering node-controller latency, controller-controller latency, load balancing among controllers and node failure.

Discrete optimization refers to the optimal solutions in a finite or countably infinite set of potential solutions. These algorithms reformulate the problem as an integer-based optimization problem which can then be solved with a conventional ILP solver such as CPLEX. In [15], mobile sinks are employed to alleviate the problem of nodes' battery depletion closer to sinks. This way distributes the traffic load among all sensors and increases network lifetime. This work aimed at finding the best way to relocate sinks inside buildings by determining their optimal locations and the duration of their sojourn time. It presents a Mixed Integer Linear Programming (MILP) formulation for finding optimal positioning of mobile sinks.

Approximation algorithms are those heuristic algorithms that find a nearly optimal solution within a reasonable execution time. This is a way of dealing with NP-completeness for optimization problem without guaranteeing to achieve the optimal solution. The goal of an approximation algorithm is to come as close as possible to the optimum value in a reasonable amount of time which is at most polynomial time. In [16], a mathematical model to determine sink locations is introduced to minimize average communication distances. The authors proposed two sink deployment algorithms; (i) global and (ii) one-hop algorithms. The global algorithm uses global information of sensors' locations, and the one-hop algorithm requires one-hop away sensor nodes information at each sink. In [17], a Simulated Annealing (SA) heuristic is used to solve the dynamic controller provisioning problem within a Wide Area Network (WAN). The basic idea was to enable or disable links live on a network to a controller from a pool of switches. In [18], the authors proposed a discrete optimization scheme based on artificial bee colony algorithm to solve the optimal sensor placement problem after transforming it into an integer optimization problem. In [19], the authors study the optimal sink deployment problem based on the grid. An efficient gridbased algorithm is developed to solve the problem through dividing the continuous search space into a finite number of discrete intersections. In this work, both clustering and routing are considered, where clusters are selected from deployed sensors. An approximation greedy algorithm is proposed to solve the problem.

Browsing the related works reveals that none of the solutions target the problem of multiple sink and multiple controller placement while considering real-time requirements in IIoT systems.

\section{PROBLEM DESCRIPTION}

In this paper, we assume an SDN-enabled sensor network [20], where sensor nodes collect information and forward it towards sink nodes. We assume existence of multiple sinks to improve the quality of service, and multiple SDN controllers to make the network more agile and flexible. However, unlike [7], instead of having fixed sink nodes, a set of mobile sink nodes are considered to forward the sensory measurements to the core network towards a Gateway. Fig. 1 illustrates such a network, where sensor nodes construct a mesh network connecting to multiple mobile sinks and controllers. Mobile sinks perform a dual role as they must receive the collected information from the sensor nodes and then after a simple data aggregation, forward it to the Gateway.

We assume that mobile sinks receive the raw data and perform the aggregation locally only when they are situated in an aggregation point. Hence, the mobile sinks must stay for a specific amount of time to collect all measurements and to perform the aggregation process. The aggregated data is then forwarded to the Gateway while the sink node is moving from one aggregation point to another. The connection to the Gateway is available along the entire mobile sink trajectory.

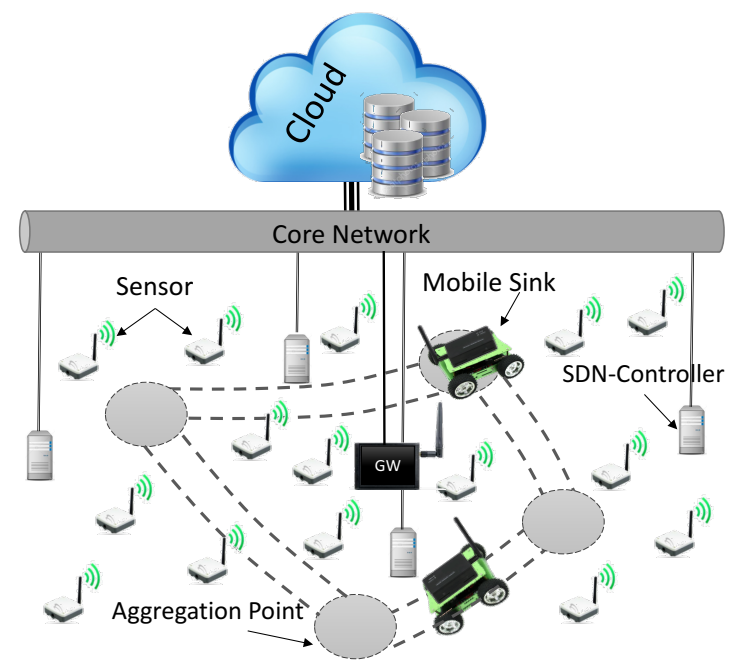

Fig. 1: Network topology.

In this section, we formalize the problem of finding the minimum number of mobile sinks and their route planning by modeling it as an optimization problem, particularly as an ILP problem, while introducing our main assumptions. Developing an ILP model for the problem enables using various wellknown ILP solver tools (e.g., CPLEX [21]), and also applying other meta-heuristic algorithms to solve the optimization problem; such as the Branch and Bound algorithm $A^{*}$, Genetic Algorithms, and Ant Colony Optimization. In other words, by transforming the problem into an ILP problem, it can be addressed with various off-the-shelf techniques.

\section{A. Problem modeling}

An IIoT system is represented by an undirected graph $G=(V, E)$, where vertices are partitioned into a set of sensors $\Gamma$, controllers $C$ and aggregation points $\Lambda$. Hence, in the graph representation of an IIoT, $V=\Gamma \cup C \cup \Lambda$. We calculate the set of $\Lambda=\left\{\lambda_{1}, \lambda_{2}, \ldots, \lambda_{M}\right\}$ based on the algorithm proposed in [7], where the optimal number and location of aggregation points are determined. An edge of the graph, $e_{i j}$ indicates a wireless connection between a pair of nodes $(i, j)$. The total number of sensors is $N$; i.e., $N=|\Gamma|$. A pair of nodes connected with an edge are called, neighbor nodes. A path with the length $l$ from node $v$ to $v^{\prime}$ is a sequence of nodes $v, v_{1}, v_{2}, \ldots, v_{l}$-where $v_{l}=v^{\prime}$; connecting node $v$ to $v^{\prime}$, such that $v_{i}, v_{i+1}$ are neighbors. Furthermore, to be able to represent our 
problem, we define $A_{S}$, denoting a set of all candidate mobile sinks. By applying the optimization algorithm, a subset of these candidate mobile sinks $S$ will be chosen, i.e. $S \subseteq A_{S}$. A mobile sink collects data upon arrival at an aggregation point. A mobile sink can visit multiple aggregation points during a certain time interval $[0, T]$, where $T$ is the hyper period of the whole system calculated by $\operatorname{LCM}\left(T_{1}, T_{2}, \ldots, T_{N}\right)^{1}$, where $T_{i}$ indicates the maximum acceptable waiting time for the $i^{t h}$ sensor. Indeed, $T_{i}$ is equal to MIN(deadline of sensor $i$, maximum buffering time of the $i^{t h}$ sensor). The reason to consider hyper period is to ensure that if a static routing mechanism is valid for one hyper period, then it is also valid for the whole run-time duration. For the sake of simplicity, in this paper, we assume that all the sensors have the same maximum acceptable waiting time, $T=T_{1}=\ldots=T_{N}$.

A sensor is said to be sink-covered in the time period of $[0, T]$, if and only if: (i) having at least one path with length $\leq l_{\max }$ to one of the aggregation points, and (ii) residing at least one mobile sink in time interval $[0, \mathrm{~T}]$ in that aggregation point. If a sensor is not sink-covered, it is assumed to be uncovered. Generally, a sensor is $k$-sink-covered if and only if it has at least $k$ paths of length $\leq l_{\max }$ to $k$ aggregation points where each of which is visited by at least one mobile sink in the time inteval $[0, T]$ ( $k$ is an integer number $\geq 1)$. We define an IIoT system as $k$-sink-covered if each sensor $i \in \Gamma$ is $k$-sink-covered.

Load balancing assumption. We assume that during runtime SDN controllers provide a load balancing mechanism where the data coming from sensors is uniformly distributed among the covering aggregation points. We define the load of sensor $i$ as the rate of data generated by the sensor per second, denoted by $\omega_{i}$. By assuming a uniform distribution of the workload among the aggregation points, each sink visiting an aggregation point only needs to be able to handle the dedicated portion of the workload. If a sensor is covered by $n$ aggregation points (i.e., the distance between those $n$ sinks and the sensor is shorter than $l_{\max }$ ), the load of the $i^{\text {th }}$ sensor on the $j^{\text {th }}$ sink covering the sensor is denoted by $w_{i, j}$, which is equal to $\omega_{i} / n$. Fig. 2 illustrates how the load is distributed among $n$ aggregation points. In the following section, the workload constraint will be formally defined.

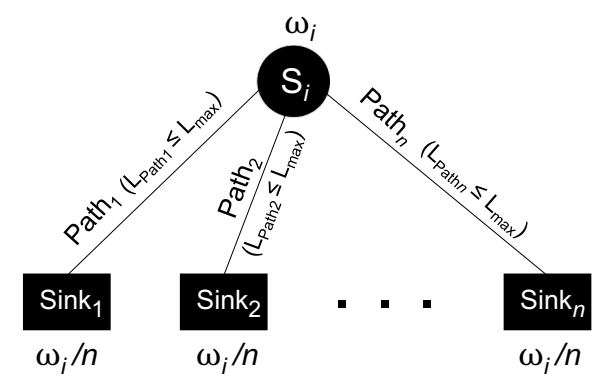

Fig. 2: Distribution of sensor measurements (load) between selected sink nodes.

\section{B. Problem Formulation}

1) Mobile Sink deployment formulation: First of all, let us introduce the notations used in the problem of finding the

\footnotetext{
${ }^{1}$ Least Common Divider
}

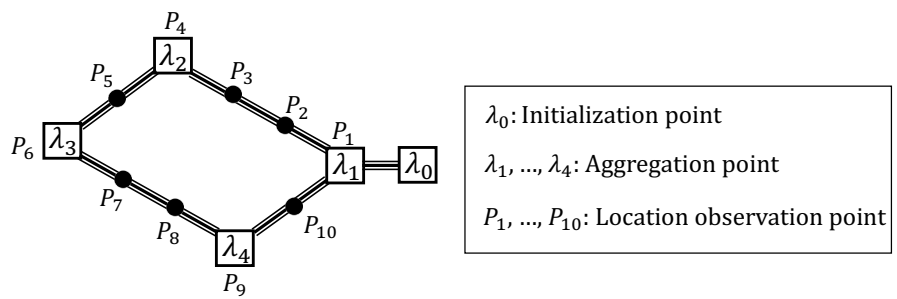

Fig. 3: An example of sink trajectory in a network with four possible aggregation points.

minimum number of mobile sinks and defining their routing plan for an IIoT system.

$$
X_{l}= \begin{cases}1 & \text { if the mobile sink } l \text { is chosen } \\ 0 & \text { else }\end{cases}
$$

The binary array $X$ determines whether a mobile sink is chosen.

$$
Y_{i, j}= \begin{cases}1 & L^{*}\left(v_{i}, \lambda_{j}\right) \leq l_{\max } \\ 0 & \text { else }\end{cases}
$$

In order to represent the k-sink-covered constraint, we define the binary matrix $Y$ that determines if the shortest distance between the $i^{\text {th }}$ sensor and the $j^{\text {th }}$ aggregation point is shorter than $l_{\text {max }}$. Indeed, $L^{*}\left(v_{i}, \lambda_{j}\right)$ is the shortest path between sensor node $v_{i}$ and the aggregation point $\lambda_{j}$, which can be calculated by the Dijkstra algorithm in terms of number of hops.

Consider $\Delta_{S_{l}, t}$ represents the location of mobile sink $S_{l}$ at time $t$. Assuming that the current location of mobile sink is known, it is possible to find a set of possible next locations $(\rho)$ for each mobile sink at any instance of time. Fig. 3 shows a simple sink trajectory for a network with four aggregation points. For the sake of simplicity, we consider that all mobile sinks are homogeneous, having the same speed, and the distance between two neighboring aggregation points is represented by the time it takes for a mobile sink traveling from one aggregation point to another. For example, it takes $2 \times t$ for a mobile sink to move from $\lambda_{2}$ to $\lambda_{3}$, where $t$ is the time granularity by which we observe the location of a mobile sink. Accordingly, we can mark all the possible mobile sink locations, illustrated by $P$ at Fig. 3. Assuming that a mobile sink is located at $\lambda_{2}$ which is the location point $P_{4}$, the set $\rho=\left\{P_{3}, P_{4}, P_{5}\right\}$ is constructed as its next locations. Thus, $\Delta_{S_{l}, t}$ is updated as follows:

$\Delta_{S_{l}, t}=\left\{\begin{array}{lr}\rho\left(\Delta_{S_{l}, t-1}\right) & \text { if }\left\{\Delta_{S_{l}, t-1}=\lambda \text { and } \Delta_{S_{l}, t-2}=\Delta_{S_{l}, t-1}\right\} \\ \Delta_{S_{l}, t-1} & \text { if }\left\{\Delta_{S_{l}, t-1}=\lambda \text { and } \Delta_{S_{l}, t-2} \neq \Delta_{S_{l}, t-1}\right\} \\ \rho\left(\Delta_{S_{l}, t-1}\right) \backslash \Delta_{S_{l}, t-2} \quad \text { else }\end{array}\right.$

A mobile sink needs to stay at an aggregation point at least $t$ time unit to collect the data from the sensor nodes. Eq. 3 implies that if a mobile sink node $S_{l}$ is located at an aggregation point $\lambda$ and has stayed there for at least one time unit $t$, then it can move towards all the possible routes that connects it to a new aggregation point including the current location. But if the stay time is less than $t$ time unit, then 
it has to stay at the current location to collect all sensor measurements. Finally, if a mobile sink node is traveling from an aggregation point to another one, it should not change its direction along the way before reaching to the intended aggregation point.

Another important constraint that must be considered is to enforce all mobile sinks to be at the initialization point $\lambda_{0}$ at the end of each hyper period of $T$. This assures that the timing requirement of the application is satisfied if there is a solution for the mobile sink deployment during only one hyper period $T$. This constraint can be formulated as below:

$$
\sum_{\forall S_{l} \in A_{S}} X_{l} Z\left(\Delta_{S_{l}, T}, \lambda_{0}\right) \geq X_{l}
$$

where, $Z()$ is a simple mapping function that shows the occupancy status of the aggregation point $\lambda_{j}$ by the $\operatorname{sink} S_{l}$ at a certain time $t$ and can be represented by:

$$
Z\left(\Delta_{S_{l}, t}, \lambda_{j}\right)= \begin{cases}1 & \text { if } \Delta_{S_{l}, t}=\lambda_{j} \\ 0 & \text { else }\end{cases}
$$

This constraint can be represented as below:

$$
P_{1}(X)=X_{l}-\sum_{\forall S_{l} \in A_{S}} X_{l} Z\left(\Delta_{S_{l}, t}, \lambda_{0}\right) \leq 0
$$

Since the location of aggregation points have been computed by the algorithm in [7] such that each sensor is definitely covered by at least $k$ aggregation points, the presence of at least one mobile sink in each aggregation point $\lambda_{i}$ ensures the k-covered-constraint. Therefore,

$$
\sum_{t=0}^{T} \sum_{\forall S_{l} \in A_{S}} X_{l} Z\left(\Delta_{S_{l}, t}, \lambda_{j}\right) \geq 1 \quad \forall \lambda_{j} \in \Lambda
$$

The $k$-sink-covered constraint for all sensors can be represented by a single formula as below:

$$
P_{2}(X)=\sum_{\forall \lambda_{j} \in \Lambda} \operatorname{Max}\left\{0,\left(1-\sum_{t=0}^{T} \sum_{\forall S_{l} \in A_{S}} X_{l} Z\left(\Delta_{S_{l}, t}, \lambda_{j}\right)\right\} \leq 0\right.
$$

However, Eq. 8 cannot guarantee that $k$ distinct mobile sinks will cover a sensor node $v_{i}$ during $[0, T]$ time period. It is especially important for ensuring the reliability requirement of the network to tolerate sink failures, otherwise if the same mobile sink visits all the aggregation points covering a sensor in a back and forth fashion during the hyper period $T$, then failure of the mobile sink can lead to network breakdown. Accordingly, we need to integrate another constraint into the ILP model as below to ensure that during the time interval $[0, \mathrm{~T}]$ at least $k$ distinct mobile sinks serve each sensor:

$$
\sum_{\forall S_{l} \in A_{S}} X_{l}\left(\min \left\{1, \sum_{t=0}^{T} \sum_{\forall \lambda_{j} \in \Lambda} Y_{i, j} Z\left(\Delta_{S_{l}, t}, \lambda_{j}\right)\right\}\right) \geq k \quad \forall v_{i} \in \Gamma
$$

This equation implies that the number of distinct selected mobile sinks visiting at least one of the aggregation points covering sensor $i$ should be equal or greater than $K$. To reflect this constraint for all sensors with a single equation, it can be rewritten as:

$$
P_{3}(X)=\sum_{v_{i} \in \Gamma} \max (0, k-E) \leq 0
$$

where, $E$ is defined as:

$$
E=\sum_{\forall S_{l} \in A_{S}} X_{l}\left(\min \left\{1, \sum_{t=0}^{T} \sum_{\forall \lambda_{j} \in \Lambda} Y_{i, j} Z\left(\Delta_{S_{l}, t}, \lambda_{j}\right)\right\}\right)
$$

Let us assume that $W_{S_{l}}$ shows the maximum workload that can be handled by a sink within an acceptable time and $\omega_{i}$ being the amount of workload imposed by a sensor node during $[0, T]$ time period. We consider that mobile sinks are able to transmit their collected measurements from sensors to the Gateway while moving from one aggregation point to another. So, mobile sinks always enter the aggregation point with full capacity.

Assuming the arbitrary inter-arrival time between two consecutive mobile sink visits, the accumulated workload at the aggregation point is $\omega_{i} /\left(t_{C}-t_{L}\right)$, where, $\left\langle t_{L}, t_{C}>\in A_{\lambda_{j}}\right.$ is a 2tuple that $t_{L}$ represent the last time a mobile sink was presented at an aggregation point $\lambda_{j}$ and $t_{C}$ is the time in which a new mobile sink has arrived at the same aggregation point. $A_{\lambda_{j}}$ is a set of all possible 2-tuples that may happen during one hyper period of $T$ and can be represented by:

$$
A_{\lambda_{j}}=\left\{<t_{L}, t_{C}>: 1 \leq t_{C} \leq T, 0 \leq t_{L}<t_{C}, \text { Condition } 1\right\}
$$

where, Condition 1 is formulated as follows:

$$
\begin{aligned}
\text { Condition } 1 & =\max \left\{0,\left(1-\sum_{\forall S_{l} \in A_{S}} X_{l} Z\left(\Delta_{S_{l}, t_{L}}, \lambda_{j}\right)\right\}\right. \\
& +\max \left\{0,\left(1-\sum_{\forall S_{l} \in A_{S}} X_{l} Z\left(\Delta_{S_{l}, t_{C}}, \lambda_{j}\right)\right\}\right. \\
& +\sum_{t_{L}<t_{i}<t_{C}} \sum_{\forall S-l \in A_{S}} X_{l} Z\left(\Delta_{S_{l}, t_{i}}, \lambda_{j}\right) \leq 0
\end{aligned}
$$

The first two terms in Condition 1 indicate that at least one mobile sink must have been occupied the aggregation point $\lambda_{j}$ at the exact $t_{L}$ and $t_{C}$ time respectively, and the third term ensures that no mobile sink has been presented at that specific aggregation point $\lambda_{j}$ during $\left(t_{L}, t_{C}\right)$. Considering all these conditions, the workload constraint can be then reflected by:

$$
\sum_{\forall v_{i} \in \Gamma} Y_{i, j} \frac{\omega_{i} /\left(t_{C}-t_{L}\right)}{\sum_{\forall \lambda_{p} \in \Lambda} Y_{i, p}} \leq W_{S_{l}} /(k-1) \quad \forall \lambda_{j} \in \Lambda
$$

where $\sum_{\forall \lambda_{p} \in \Lambda} Y_{i, p}$ is the number of aggregation points that cover the $i^{\text {th }}$ sensor. Due to the reliability requirement, we apply a more strict workload constraint; if $k-1$ sinks fail, the remaining sink should be able to handle the entire workload of all the failed sinks without any violation of latency requirements. This is the reason behind having the right side of the inequality divided by $k-1$. The load constraint for all sinks can be formulated by:

$$
P_{4}(X)=\sum_{\lambda_{j} \in \Lambda} \max \left\{0,\left(\sum_{\forall v_{i} \in \Gamma} \frac{Y_{i, j} \omega_{i} /\left(t_{C}-t_{L}\right)}{\sum_{\forall \lambda_{t} \in \Lambda} Y_{i, t}}\right)-\frac{W_{S_{l}}}{k-1}\right\} \leq 0
$$


2) Optimization problem formulation: The optimization problem is then formulated as follows:

$$
\begin{gathered}
\text { Minimize: } \quad \operatorname{TotalCost}(X)=\sum_{\forall S_{l} \in A_{S}} X_{l} \operatorname{Cost}_{l}, \\
\text { Subject to: }(6),(8),(10),(15) .
\end{gathered}
$$

Now we have modeled the problem as an ILP problem, particularly as a Combinatorial Optimization Problem (COP), a subset of the ILP category, where the domain of optimization parameters is only 0,1 .

3) Fitness function: In the context of combinatorial optimization problems, it is highly desirable to integrate the goal function and all constraints into one function that can be used as a fitness function, particularly when meta-heuristic algorithms are going to be applied. Thus, we suggest a single fitness function comprising the whole optimization problem.

$$
\text { Fitness }(X)=\text { TotalCost }(X)+\beta\left(\sum_{i=1}^{4} P_{i}(X)\right)
$$

Functions $P_{1}$ to $P_{4}$ are penalty functions applied to measure satisfiability of a given assignment in terms of all four constraints. When all the penalty functions for a given assignment are equal to zero, it means that the given assignment meets all the constraints; otherwise (i.e., when it is greater than zero), at least one constraint is violated by the given assignment. $\beta$ is a penalty coefficient used to guide the search towards valid solutions. The coefficient tunes the weight of the penalty functions with regards to the range of the goal function.

\section{DISCUSSION AND CONCLUSION}

In this paper, we first investigated the challenges to employ multiple mobile sinks for data collection in an IIoT system. We discussed four main challenges to address the deployment of mobile sinks to (i) find optimal number and locations of aggregation points, (ii) specify an effective trajectory to connect the aggregations points, (iii) optimal routing of mobile sinks along the trajectory, (iv) find the minimum number of mobile sinks. Among these elements, this paper particularly focused on two latter challenges. We proposed an ILP model for both static routing of multiple mobile sinks and minimizing the number of required mobile sinks in an IIoT system while respecting reliability and timing requirements. In such a complex optimization problem, presenting an ILP model constitutes the key part of addressing the problem, since afterward there are a large number of existing ILP solvers, which can be easily applied to solve the ILP problem. Therefore, this paper creates the foundation for the deployment of mobile sinks in IIoT systems by presenting an analytical model of the problem.

For future, we intend to apply CPLEX ILP solver to address the ILP problem. We will also examine the total deployment cost of the system, where we use mobile sinks in comparison with conventional WSNs using fixed sink nodes. Using only fixed sink nodes means that all the aggregation points are filled by a fixed sink node. This tradeoff significantly depends on two factors including the number of required mobile sinks, and the cost difference between fixed sink nodes and mobile sinks. For the sake of completeness, we will also investigate the impact of using mobile sinks on the performance and functionality of the system at run-time whereas the scope of this paper was to reduce deployment cost of the system considered at the design-time of the system.

\section{REFERENCES}

[1] S. Jeschke, C. Brecher, T. Meisen, D. Özdemir, and T. Eschert, "Industrial internet of things and cyber manufacturing systems," in Industrial Internet of Things. Springer, 2017, pp. 3-19.

[2] O. Gnawali, R. Fonseca, K. Jamieson, D. Moss, and P. Levis, "Collection tree protocol," in Proceedings of the 7th ACM conference on embedded networked sensor systems. ACM, 2009, pp. 1-14.

[3] M. Doudou, D. Djenouri, N. Badache, and A. Bouabdallah, "Synchronous contention-based mac protocols for delay-sensitive wireless sensor networks: A review and taxonomy," Journal of Network and Computer Applications, vol. 38, pp. 172-184, 2014.

[4] K.-H. Phung, B. Lemmens, M. Goossens, A. Nowe, L. Tran, and K. Steenhaut, "Schedule-based multi-channel communication in wireless sensor networks: A complete design and performance evaluation," Ad Hoc Networks, vol. 26, pp. 88-102, 2015.

[5] Z. Zhou, C. Du, L. Shu, G. Hancke, J. Niu, and H. Ning, "An energybalanced heuristic for mobile sink scheduling in hybrid wsns," IEEE Transactions on Industrial Informatics, vol. 12, no. 1, pp. 28-40, 2016.

[6] E. Cardarelli, V. Digani, L. Sabattini, C. Secchi, and C. Fantuzzi, "Cooperative cloud robotics architecture for the coordination of multiagv systems in industrial warehouses," Mechatronics, vol. 45, pp. 1-13, 2017.

[7] H. R. Faragardi, H. Fotouhi, T. Nolte, and R. Rahmani, "A cost efficient design of a multi-sink multi-controller wsn in a smart factory," in Proceedings of the 19th IEEE International Conference on High Performance Computing and Communications (HPCC). IEEE, 2017.

[8] K. Ahmed, J. O. Blech, M. A. Gregory, and H. Schmidt, "Software defined networking for communication and control of cyber-physical systems," in Parallel and Distributed Systems (ICPADS), 2015 IEEE 21 st International Conference on. IEEE, 2015, pp. 803-808.

[9] C. Tunca, S. Isik, M. Y. Donmez, and C. Ersoy, "Distributed mobile sink routing for wireless sensor networks: A survey," IEEE communications surveys \& tutorials, vol. 16, no. 2, pp. 877-897, 2014.

[10] J. Luo, J. Panchard, M. Piorkowski, M. Grossglauser, and J.-P. Hubaux, "Mobiroute: Routing towards a mobile sink for improving lifetime in sensor networks," in DCOSS, vol. 6. Springer, 2006, pp. 480-497.

[11] J. Shi, X. Wei, and W. Zhu, "An efficient algorithm for energy management in wireless sensor networks via employing multiple mobile sinks," International Journal of Distributed Sensor Networks, vol. 12, no. 1, p. 3179587, 2016.

[12] J. Tang, B. Hao, and A. Sen, "Relay node placement in large scale wireless sensor networks," Computer communications, vol. 29, no. 4 , pp. 490-501, 2006.

[13] B. Heller, R. Sherwood, and N. McKeown, "The controller placement problem," in Proceedings of the first workshop on Hot topics in software defined networks. ACM, 2012, pp. 7-12.

[14] D. Hock, S. Gebert, M. Hartmann, T. Zinner, and P. Tran-Gia, "Pocoframework for pareto-optimal resilient controller placement in sdn-based core networks," in Network Operations and Management Symposium (NOMS). IEEE/IFIP, 2014, pp. 1-2.

[15] L. B. Saad and B. Tourancheau, "Towards an optimal positioning of multiple mobile sinks in wsns for buildings," International Journal on Advances in Intelligent Systems, vol. 2, no. 4, pp. 411-421, 2009.

[16] Z. Vincze, R. Vida, and A. Vidacs, "Deploying multiple sinks in multihop wireless sensor networks," in IEEE International Conference on Pervasive Services,. IEEE, 2007, pp. 55-63.

[17] M. F. Bari, A. R. Roy, S. R. Chowdhury, Q. Zhang, M. F. Zhani, R. Ahmed, and R. Boutaba, "Dynamic controller provisioning in software defined networks," in Proceedings of the 9th IEEE International Conference on Network and Service Management (CNSM). IEEE, 2013, pp. $18-25$.

[18] H. Sun and O. Buyukozturk, "Optimal sensor placement in structural health monitoring using discrete optimization," Smart Materials and Structures, vol. 24, no. 12, p. 125034, 2015.

[19] Y. Gu, Y. Ji, J. Li, H. Chen, B. Zhao, and F. Liu, "Towards an optimal sink placement in wireless sensor networks," in Proceeding of IEEE International Conference on Communication (ICC). IEEE, 2010, pp. $1-5$.

[20] L. Galluccio, S. Milardo, G. Morabito, and S. Palazzo, "Sdn-wise: Design, prototyping and experimentation of a stateful sdn solution for wireless sensor networks," in Computer Communications (INFOCOM), 2015 IEEE Conference on. IEEE, 2015, pp. 513-521.

[21] IBM, "Cplex optimizer," En ligne]. Available: http://www01.ibm.com/software/commerce/optimization/cplex-optimizer, 2012. 